\title{
CALIBRAÇÃO DE MODELO PARA A SIMULAÇÃO DE VAZÃO E DE FÓSFORO TOTAL NAS SUB-BACIAS DOS RIOS CONRADO E PINHEIRO - PATO BRANCO (PR) ${ }^{(1)}$
}

\author{
Valmir Baltokoski ${ }^{(2)}$, Maria Hermínia Ferreira Tavares ${ }^{(3)}$, Ronalton Evandro \\ Machado $^{(4)} \&$ Marcio Paulo de Oliveira ${ }^{(5)}$
}

\begin{abstract}
RESUMO
O desenvolvimento de modelos hidrológicos capazes de predizer o impacto de fontes difusas de poluição e do uso e ocupação do solo na qualidade das águas superficiais e subterrâneas tem auxiliado o estudo de agroecossistemas. Com esse objetivo, foi utilizado o modelo SWAT 2005 (Soil and Water Assessment Tool) para avaliar sua sensibilidade na predição da vazão e do fluxo de massa do $P$ total. $O$ estudo foi realizado em duas microbacias hidrográficas contíguas, dos rios Conrado e Pinheiro, afluentes do rio Pato Branco, localizadas nos municípios de Pato Branco e Mariópolis, no Estado do Paraná. Foram utilizados dados climatológicos do período 1979/2006 e dados observados de vazão e concentração de $P$ total dos anos 2004/2005 de duas estações de monitoramento, localizadas na parte inferior do curso principal dos rios Conrado e Pinheiro. Utilizou-se a interface AvSWAT_X, com o SIG ArcView 3.3 ${ }^{\circledR}$ e a extensão Spatial Analyst 2.0®, para entrada e manipulação dos dados. As médias anuais e mensais observadas de vazão e $P$ total foram comparadas aos dados simulados. O Coeficiente de Eficiência de NashSutcliffe (COE) foi utilizado para avaliar a eficiência do modelo. A modelagem foi melhorada com a associação de análise de sensibilidade, autocalibração e calibração manual, verificando-se que, com frequência de amostragem regular, o modelo SWAT 2005 realizou de forma aceitável as simulações de vazão e de exportação de $P$ total. Já com frequência de amostragem irregular e pequeno número de dados, os procedimentos de análise de sensibilidade e de autocalibração não foram eficientes na calibração do modelo SWAT 2005 para a simulação de vazão e
\end{abstract}

\footnotetext{
(1) Parte da Dissertação de Mestrado do primeiro autor junto ao Programa de Pós-Graduação em Engenharia Agrícola da Universidade Estadual do Oeste do Paraná - UNIOESTE. Recebido para publicação em novembro de 2008 e aprovado em novembro de 2009.

${ }^{(2)}$ Mestre em Recursos Hídricos e Saneamento Ambiental. Av. XV de Novembro 246, CEP 85570-000 São João (PR). E-mail: vbaltokoski@gmail.com

(3) Professora Doutora, Programa de Pós-Graduação em Engenharia Agrícola, Universidade Estadual do Oeste do Paraná UNIOESTE. Rua Universitária 2069, Bairro Faculdade, CEP 85819-110 Cascavel (PR). E-mail: mhstavar@gmail.com

(4) Professor Doutor, Departamento de Engenharia Ambiental, Escola de Engenharia de Piracicaba - EEP. E-mail: remachad@yahoo.com.br

(5) Mestrando, Programa de Pós-Graduação em Engenharia Agrícola, UNIOESTE. Bolsista Capes. E-mail:marciopaulobr@hotmail.com
} 
exportação de $\mathbf{P}$ total. Foram encontrados diferentes níveis de sensibilidade entre as duas estações, refletindo as desigualdades entre as Unidades de Resposta Hidrológica. A distribuição mensal simulada das exportações de $\mathbf{P}$ mostrou a heterogeneidade da aplicação do nutriente ao longo do ano.

Termos de indexação: geotecnologias, modelagem hidrológica, poluição difusa, qualidade da água, transporte de fósforo.

\title{
SUMMARY: MODEL CALIBRATION FOR FLOW RATE AND TOTAL PHOSPHOROUS EXPORT SIMULATIONS IN THE WATERSHEDS OF THE RIVERS CONRADO AND PINHEIRO, PATO BRANCO $(P R)$
}

\begin{abstract}
Hydrological models developed to predict the impacts of non-point source pollution and land use and occupation on water quality have been extremely useful in the study of agricultural systems. For this purpose, the objective of this study was to evaluate the sensitivity of the model SWAT 2005 (Soil and Water Assessment Tool) to simulate the flow rate and total phosphorous flow mass. The research was conducted in two contiguous watersheds of the Conrado and Pinheiro rivers, affluents to the Pato Branco river, in Pato Branco and Mariopolis counties, state of Paraná. Climatological data of the period 1979-2006 and observation data of flow rate and total phosphorous export of the years 2004 and 2005 from two weather stations (in the lower part of the main water course of the Conrado and Pinheiro rivers) were used. The model requires input data in spatial format of land use and soil data, associated to a digital elevation model (DEM). The AvSWAT_Xinterface was used with ArcView 3.3® and its extension Spatial Analyst $2.0{ }^{\circledR}$, for data input and manipulation. Monthly and yearly averages of flow rate and total phosphorous export were compared to the observed values. The Nash-Sutcliffe Coefficient (COE) was used to evaluate the modeling efficiency. The modeling was improved by including the sensitivity analysis, autocalibration tool and manual adjustment, showing that, with regular sampling frequency, the SWAT 2005 model simulated the flow rate and total phosphorous export satisfactorily. In the case of irregular sampling frequency and limited dataset, however, autocalibration and sensitivity analysis were not efficient to simulate the flow rate and total phosphorous export results. Besides, different sensitivity levels were observed for the two stations, reflecting inequalities of the Hydrologic Response Units. The simulated monthly distribution of phosphorous export showed the heterogeneity of Papplication over the course of the year.
\end{abstract}

Index terms: geotechnologies, hydrologic modeling, nonpoint source pollution, water quality, phosphorous transport.

\section{INTRODUÇÃO}

As fontes difusas de poluição geram os maiores problemas ambientais encontrados nas bacias hidrográficas agrícolas. Assim, o conhecimento da origem espacial das cargas geradoras de impactos negativos é importante para a gestão dessas bacias. A modelagem ambiental apresenta-se como ferramenta eficiente na tarefa de estudar e entender grande parte dos processos físicos e químicos que ocorrem no ambiente delimitado geograficamente.

Com o desenvolvimento da técnica da computação surgiram diversos modelos hidrológicos e de qualidade de água, os quais geralmente exigem grande volume de dados de entrada, cujos arquivos são criados por sistemas geográficos de informações e suas interfaces. Entre os modelos, destaca-se o SWAT (Soil and Water
Assessment Tool): desenvolvido na década de 1990, ele é baseado em processos e é contínuo no tempo, considerando clima, hidrologia, erosão, sedimentação, crescimento de plantas, nutrientes, pesticidas e manejo agrícola. Empregando parâmetros distribuídos, o SWAT tem sido amplamente utilizado com o objetivo de simular os processos que ocorrem no ambiente, buscando entender as interações, identificar a origem das contaminações, prever o que poderá acontecer naquele cenário, a fim de estabelecer as causas e efeitos dos impactos provocados pelas fontes difusas de poluição (Arnold \& Fohrer, 2005). Também tem sido empregado em conjunto com o método TMDL (Total Maximum Daily Load), como forma de predizer a evolução da qualidade da água em uma bacia hidrográfica, bem como para determinar ações adequadas de conservação, monitoramento e recuperação (Bittencourt \& Gobbi, 2006). 
Para Machado \& Vettorazzi (2003), o SWAT permite que diferentes processos físicos sejam simulados na bacia hidrográfica, para analisar os impactos das alterações no uso do solo sobre o escoamento superficial e subterrâneo, a produção de sedimentos e a qualidade da água. Para atingir esses objetivos, o modelo: baseia-se em características físicas da bacia; usa dados de entrada normalmente disponíveis; opera de maneira eficiente sobre médias e grandes bacias; e permite realizar simulações para longos períodos (> 100 anos).

Na calibração do modelo, são realizados testes com parâmetros de entrada conhecidos e as saídas são usadas para ajustar ou estimar parâmetros e variáveis. Na versão SWAT 2005, o processo de calibração pode ser automatizado, sendo chamado de Autocalibração (Green \& Griensven, 2008). O processo geralmente é precedido pela Análise de Sensibilidade, a qual gera um conjunto de arquivos com as informações que serão utilizadas na autocalibração (Green \& Griensven, 2008). A calibração também pode ser realizada manualmente, alterando-se cada parâmetro que possa ter influência para a variável em ajuste. Nesse caso, altera-se um parâmetro por vez e executa-se o modelo para verificar as mudanças nos valores de saída, repetindo o processo até que um ajuste aceitável seja obtido (Neitsch et al., 2007).

Segundo Griensven (2007), os procedimentos de calibração e validação do modelo devem seguir a ordem: processos hidrológicos, aporte de sedimentos e qualidade da água. Biesbrouck \& Wyseure (2002) salientam que, para calibrar o balanço hídrico e o escoamento fluvial, é necessário conhecer as condições reais que ocorrem na bacia.

O objetivo deste trabalho foi realizar a calibração do modelo SWAT 2005 para a simulação da vazão e exportação de $\mathrm{P}$ total nas sub-bacias dos rios Conrado e Pinheiro, localizados nos municípios de Pato Branco e Mariópolis (PR).

\section{MATERIAL E MÉTODOS}

\section{Área de estudo}

A área de estudo compreendeu as sub-bacias dos rios Conrado e Pinheiro, as quais fazem parte da bacia hidrográfica do rio Pato Branco. Elas estão localizadas nos municípios de Pato Branco e Mariópolis (PR) (Figura 1), entre as latitudes de $26^{\circ} 16^{\prime} 16$ " S e $26^{\circ} 21^{\prime} 47$ " S e as longitudes: $52^{\circ} 35^{\prime} 36^{\prime}$ " W e $52^{\circ} 41$ ' 36 " W (UTM: E=331096, N=7083009 e $\mathrm{E}=340949, \mathrm{~N}=7093322)$. A sub-bacia do rio Conrado, com área de $24,11 \mathrm{~km}^{2}$, é contígua à sub-bacia do rio Pinheiro, com área de $28,86 \mathrm{~km}^{2}$, perfazendo uma área total de $52,97 \mathrm{~km}^{2}$.

$\mathrm{Na}$ área de estudo encontram-se instaladas quatro estações de monitoramento, as quais fazem parte do Programa Nacional do Meio Ambiente II (PNMA II), operacionalizado a partir de 2000. Utilizaram-se somente os dados de duas estações: RC02 (coordenadas $\mathrm{E}=337622, \mathrm{~N}=7090.056$, código ANA 65925780) e RP02 (coordenadas $\mathrm{E}=338435, \mathrm{~N}=7089.323$, código ANA 65925770), a jusante dos rios Conrado e Pinheiro, respectivamente, por estarem mais próximas do exutório das sub-bacias e por representarem melhor o fluxo do canal.

A estação RC02 apresenta dados diários de vazão do período de 4/06/2004 até 24/06/2005, totalizando 445 registros (Paraná, 2007), com valor médio de $0,51 \mathrm{~m}^{3} \mathrm{~s}^{-1}$. Da estação RP02 constam apenas 23 registros de vazão no período de 4/11/2003 a 4/01/2006 (Paraná, 2007), com periodicidade irregular e valor médio de $0,39 \mathrm{~m}^{3} \mathrm{~s}^{-1}$.

\section{Modelo empregado}

Utilizou-se o modelo de simulação hidrológica SWAT 2005, com a interface SIG ArcView 3.3® e a extensão Spatial Analyst 2.0®. Para o propósito de modelagem, o SWAT divide a bacia em sub-bacias com base nas características topográficas do terreno, preservando o canal natural. Cada sub-bacia pode ser parametrizada pelo SWAT, usando uma série de Unidades de Resposta Hidrológica (Hydrologic Response Units - HRUs), as quais correspondem a uma única combinação de cobertura da terra e classes de solo dentro da sub-bacia. Uma ou mais combinações de uso da terra e classes de solo podem ser criadas para cada sub-bacia. Um nível de sensibilidade é adotado para eliminar áreas de uso da terra menores

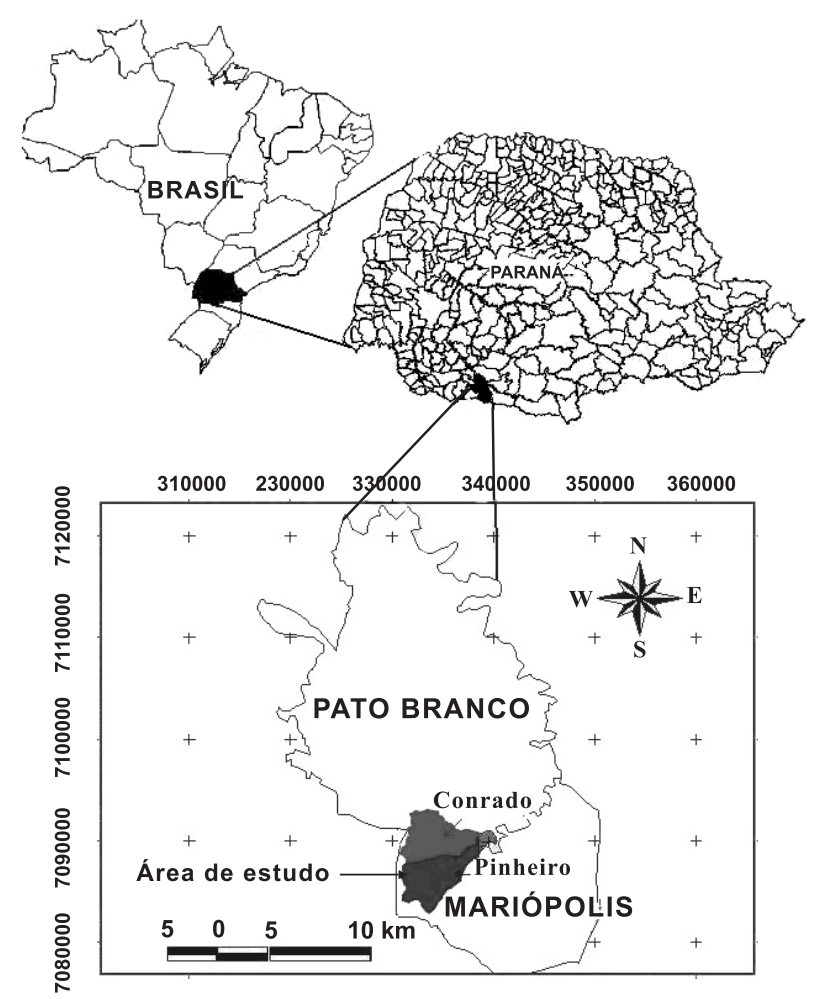

Figura 1. Localização da área de estudo. 
do que o valor arbitrado. O segundo passo controla a criação das Unidades de Resposta Hidrológica com base na distribuição dos usos da terra selecionados sobre diferentes tipos de solo. O solo que cobre uma percentagem de área dentro da área de uso da terra menor do que o nível de sensibilidade é eliminado.

Para a determinação do número de sub-bacias, o algoritmo requer três pontos conhecidos de coordenadas: $\mathrm{x}, \mathrm{y}$ e $\mathrm{z}$ (altitude), para estimar as declividades e definir os canais de drenagem (Jenson \& Domingue, 1988). O método considera, para direcionar o fluxo, as oito células vizinhas de cada célula avaliada (procedimento D8), optando por aquela de maior declividade. As direções de fluxo são calculadas pelo procedimento de direcionamento de fluxo a partir do Modelo Digital de Elevação. Esses fluxos são acumulados pelo procedimento de acumulação de fluxo, que direcionou cada célula do Modelo Digital de Elevação e conta quantas células acima contribuíram para o fluxo em cada célula.

Quanto à definição do número de sub-bacias, foram feitas simulações para oito níveis de discretização, considerando as áreas-limite de 25, 50, 100, 150, 200, 300, 400 e 500 ha. Para a geração das Unidades de Resposta Hidrológica, foi definido o limiar de $20 \%$ para o uso do solo e $10 \%$ para classe de solo, conforme sugerido em Biesbrouck \& Wyseure (2002).

Os dados para compor o Modelo Digital de Elevação (MDE) foram extraídos da carta topográfica Mariópolis MI-2862/4 da Divisão de Serviços Geográficos do Exército, escala 1:50.000, com curvas de nível com equidistância de $20 \mathrm{~m}$. O mapa de uso e ocupação do solo foi obtido a partir de imagem do satélite Landsat TM5, bandas 3, 4 e 5, de junho de 2000, com resolução espacial de $30 \mathrm{~m}$, convertida em polígonos vetoriais. $\operatorname{sh} p$, associada a um arquivo tabular em formato.$d b f$, contendo suas respectivas codificações (Quadro 1).

Cinco classes predominantes de uso do solo foram identificadas: lavoura (33,13\%), capoeira $(21,64 \%)$, mata (26,72 \%), campo (15,54 \%) e reflorestamento $(2,96 \%)$. As principais culturas de verão são soja, milho e feijão, e no inverno são cultivados aveia e trigo. A ocupação humana caracteriza-se por pequenas e médias propriedades, onde se pratica a rotação de culturas, principalmente milho, soja, feijão, com pastagens para gado de corte e produção de leite, ocupando áreas que avançam até próximo às margens dos rios (Machado, 2006).

Quatro classes predominantes de solo são encontradas na área de estudo (Machado, 2006): Latossolo (37,26 \%), Nitossolo (32,74\%), Cambissolo $(28,21 \%)$ e Neossolo (1,79 \%) (Quadro 2$)$.

Os dados climatológicos utilizados neste trabalho são da Estação Climatológica do Instituto Agronômico do Paraná (IAPAR) de Pato Branco, código ANNEL 2652035, e do Sistema Meteorológico do Paraná (SIMEPAR) de Pato Branco, código ANNEL 26075241. O modelo requer dados diários de precipitação pluvial, temperaturas máxima e mínima do ar, radiação solar, velocidade do vento e umidade relativa.

Segundo a classificação de Köppen, a região enquadra-se no tipo climático $\mathrm{Cfb}$, com precipitação pluvial média anual no período de 1979 a 2006 igual a $2.094 \mathrm{~mm}$.

A definição das fontes de incerteza foi realizada por meio de análise de sensibilidade dos parâmetros e variáveis de entrada. Adotou-se um cenário-base, considerando os anos de 2004 e 2005 e dois diferentes tipos de manejo (Quadro 3).

Quadro 1. Classes de uso original, código no AvSWAT, subclasses, código das subclasses AvSWAT e percentual de ocupação

\begin{tabular}{|c|c|c|c|c|}
\hline Classe original & Código classe - AvSWAT & Subclasse & Código subclasse - AvSWAT & Ocupação $^{(1)}$ \\
\hline Lavoura & AGRR & $\begin{array}{l}\text { Soja } \\
\text { Milho } \\
\text { Feijão } \\
\text { Aveia } \\
\text { Trigo }\end{array}$ & $\begin{array}{l}\text { SOYB } \\
\text { CORN } \\
\text { GRBN } \\
\text { OATS } \\
\text { WWHT }\end{array}$ & $\begin{array}{r}\% \\
32 \\
25 \\
5 \\
36 \\
2\end{array}$ \\
\hline Campo & PAST & $\begin{array}{l}\text { Pastagem permanente } \\
\text { Capim e similares }\end{array}$ & $\begin{array}{l}\text { PAST } \\
\text { RYEG }\end{array}$ & $\begin{array}{l}90 \\
10\end{array}$ \\
\hline Capoeira & FRST & Capoeira & $\operatorname{RNGB}^{(3)}$ & $\begin{array}{l}65 \\
20\end{array}$ \\
\hline Mata & FRSE & $\begin{array}{l}\text { Áreas baixas } \\
\text { Áreas baixas }\end{array}$ & $\begin{array}{l}\text { BROM }^{(4)} \\
- \text { BROM }^{--}\end{array}$ & $\begin{array}{l}15 \\
70 \\
30\end{array}$ \\
\hline Reflorestamento & PINE & $\cdots$ & -..- & 100 \\
\hline
\end{tabular}

(1) Para as subclasses de Lavoura, considerou-se uma estimativa média contínua ao longo do ano. ${ }^{(2)}$ Não foi criada subclasse, permanecendo como Classe. ${ }^{(3)}$ Áreas cobertas por arvoredos, ervas daninhas e gramas, com predominância de arvoredo. ${ }^{(4)}$ Área baixa, próxima a rios e coberta por grama, não roçada e geralmente cortada para alimentação animal. 
Quadro 2. Propriedades físicas por classe de solo

\begin{tabular}{|c|c|c|c|c|c|c|}
\hline \multirow[b]{2}{*}{ Solo } & \multicolumn{3}{|c|}{ Porosidade } & \multirow{2}{*}{ Densidade } & \multirow{2}{*}{$\begin{array}{l}\text { Condutividade } \\
\text { hidráulica saturada }\end{array}$} & \multirow{2}{*}{$\begin{array}{c}\text { Capacidade } \\
\text { de água disponível }\end{array}$} \\
\hline & Macro & Micro & Total & & & \\
\hline & 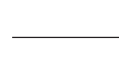 & $-\mathrm{m}^{3} \mathrm{~m}^{-3}$ & - & $\mathrm{Mg} \mathrm{m}^{-3}$ & $\mathrm{~m} \mathrm{~h}^{-1}$ & $\mathrm{~m} \mathrm{~m}^{-1}$ \\
\hline Latossolo & 6,96 & 54,78 & 61,74 & 1,17 & 0,650 & 0,14 \\
\hline Nitossolo & 11,38 & 50,77 & 62,15 & 1,02 & 0,090 & 0,17 \\
\hline Cambissolo & 4,54 & 54,73 & 59,27 & 1,25 & 0,940 & 0,07 \\
\hline Neossolo & 7,26 & 45,18 & 52,44 & 1,20 & 0,610 & 0,12 \\
\hline
\end{tabular}

Quadro 3. Uso e manejo do solo inseridos no SWAT 2005 como cenário-base

\begin{tabular}{|c|c|c|c|}
\hline \multicolumn{2}{|c|}{ Manejo - 2004: Rotação aveia/soja ${ }^{(1)}$} & \multicolumn{2}{|c|}{ Manejo - 2005: Rotação aveia/milho } \\
\hline Data & Operação & Data & Operação \\
\hline 15 de março & Colheita de soja & 15 de fevereiro & Colheita de milho \\
\hline 20 de abril & Plantio de aveia & 15 de abril & Plantio de aveia \\
\hline 20 de abril & Fertilizante cama de aviário ${ }^{(2)}$ & 15 de abril & Fertilizante cama de aviário ${ }^{(2)}$ \\
\hline 10 de setembro & Colheita da aveia & 05 de setembro & Colheita da aveia \\
\hline 15 de outubro & Plantio de soja & 10 de setembro & Plantio de milho \\
\hline 15 de outubro & Fertilizante $^{(2)}$ & 10 de setembro & Fertilizante ${ }^{(2)}$ \\
\hline
\end{tabular}

(1) Para todas as simulações e cenários, considerou-se plantio direto. ${ }^{(2)}$ Não adicionado no cenário-base.

A calibração foi realizada sem entrada de fertilizantes, objetivando introduzir aplicações de fertilizantes conforme prática agrícola da região e identificar os valores de $\mathrm{P}$ total resultantes na descarga.

\section{Avaliação estatística}

Na avaliação estatística do desempenho do modelo, os dados mensais de vazão simulados foram comparados aos dados observados nas duas estações de monitoramento.

Para Machado \& Vettorazzi (2003), um dos mais importantes critérios estatísticos para avaliar o ajuste de modelos hidrológicos é o Coeficiente de Eficiência de Nash e Sutcliffe (COE), calculado por meio da equação (1):

$$
C O E=1-\frac{\sum_{i=1}^{n}\left(E_{m}-E_{s}\right)^{2}}{\sum_{i=1}^{n}\left(E_{m}-\overline{E_{s}}\right)^{2}}
$$

em que $E_{m}$ é o evento observado; $E_{s}$, o evento simulado pelo modelo; $\bar{E}_{s}$, a média do evento observado no período de simulação; e $n$, o número de eventos. O coeficiente $(C O E)$ pode variar entre negativo infinito a 1 , sendo o valor 1 indicativo de um perfeito ajuste (Asce, 1993).
Conforme Silva et al. (2008), quando o valor de COE resultar maior que 0,75 , o desempenho do modelo é considerado bom. Para valores de $C O E$ entre 0,36 e 0,75 , o desempenho é considerado aceitável, enquanto valores de $C O E$ inferiores a 0,36 fazem com que o modelo seja julgado como inaceitável.

\section{RESULTADOS E DISCUSSÃO}

\section{Delimitação das unidades de resposta hidrológica}

A área que apresentou melhor resposta em relação às classes originais de uso e tipo de solo e que seguiu as regras para delimitação das Unidades de Resposta Hidrológica e das sub-bacias (Biesbrouck \& Wyseure, 2002) foi a de 100 ha, resultando em 175 Unidades de Resposta Hidrológica, distribuídas em 25 sub-bacias.

\section{Análise de sensibilidade e calibração do modelo para vazão}

A análise de sensibilidade e a calibração do modelo foram realizadas primeiramente para vazão na estação $\mathrm{RC} 02$.

Na versão SWAT 2005, não é possível controlar as variáveis de entrada para a análise de sensibilidade, sendo estas em número de 27, definidas por default pelo modelo (Green \& Griensven, 2007). Como 
resultado da análise, é gerado um arquivo contendo a posição dos parâmetros analisados pela função objetivo $(O F)$, com valores de 1 para a maior sensibilidade e 28 para a menor ou nenhuma sensibilidade. A autocalibração, objetivando a vazão na estação RC02, teve 5.328 iterações, resultando no Coeficiente de Nash-Sutcliffe $C O E=0,68$, considerado aceitável.

Para a estação RP02, os resultados da análise de sensibilidade para vazão foram semelhantes aos da estação RC02. A simulação realizada após a análise de sensibilidade e autocalibração na estação RP02, para o período de 1/1/2003 a 31/12/2005, resultou no valor de $C O E=0,70$, considerado aceitável.

Entretanto, a curva de distribuição temporal simulada, comparada aos dados observados, não apresentou ajuste, indicando que, com base nos 21 dados observados, os procedimentos de análise de sensibilidade e autocalibração não se mostraram suficientemente confiáveis para simular a vazão na estação RP02. Segundo Neitsch et al. (2006), condições de isolamento temporal de amostras dificilmente serão reproduzidas por modelos de longo período, como o SWAT. O trabalho de Lemonds \& Kasch (2007) descreve discrepâncias na curva de distribuição temporal de vazão com o emprego do SWAT 2005; o modelo subestimou os resultados, da mesma forma como ocorreu com Benaman et al. (2005).

\section{Análise de sensibilidade e calibração para exportação de fósforo total}

No quadro 4 encontra-se a descrição dos códigos dos parâmetros utilizados pelo SWAT 2005 e que apresentaram efeitos na presente modelagem.
Os resultados das análises de sensibilidade para as duas estações são mostrados na figura 2 .

Um total de 24 parâmetros apresentou sensibilidade, sendo os mais sensíveis: CN2 (nível 1), SOL_ORGP (nível 2) e SLOPE (nível 3). A Curva Número (CN2 para condição 2 de umidade do solo) é usada para o cálculo do escoamento superficial, sendo relacionada ao tipo de solo, ao uso da terra e às práticas de manejo (Arnold et al., 1998), fatores responsáveis pelos processos que interferem na qualidade da água. Já a variável SLOPE altera o escoamento superficial. Foram encontradas diferenças no nível de sensibilidade entre as estações RC02 e RP02, refletindo as desigualdades entre as Unidades de Resposta Hidrológica.

A sensibilidade para a concentração inicial de $\mathrm{P}$ orgânico no solo é um resultado importante, pelo fato de ser ela o principal parâmetro para auferir a qualidade da água no presente estudo.

O procedimento de autocalibração para os dados da estação RC02 resultou no $C O E=0,27$, considerado inaceitável. Procedeu-se então à calibração manual, modificando-se as variáveis: SOL_MINP (P mineral), SOL_ORGP (P orgânico), FRT_LY1 (fração de fertilizante aplicado na camada superior do solo), RSDCO (coeficiente de cobertura de resíduo de colheita), BIOMIX (coeficiente de eficiência da mistura biológica), PPERCO (coeficiente de percolação do P), PHOSKD (coeficiente de partição do P no solo) e Al2 (fração de biomassa de alga no canal) (Arnold et al., 1998; Jayakrishnan et al., 2005; Santhi et al., 2005).

A carga anual resultante da simulação para o período 2004/2005, após a calibração manual, foi de

Quadro 4. Códigos utilizados pelo SWAT 2005

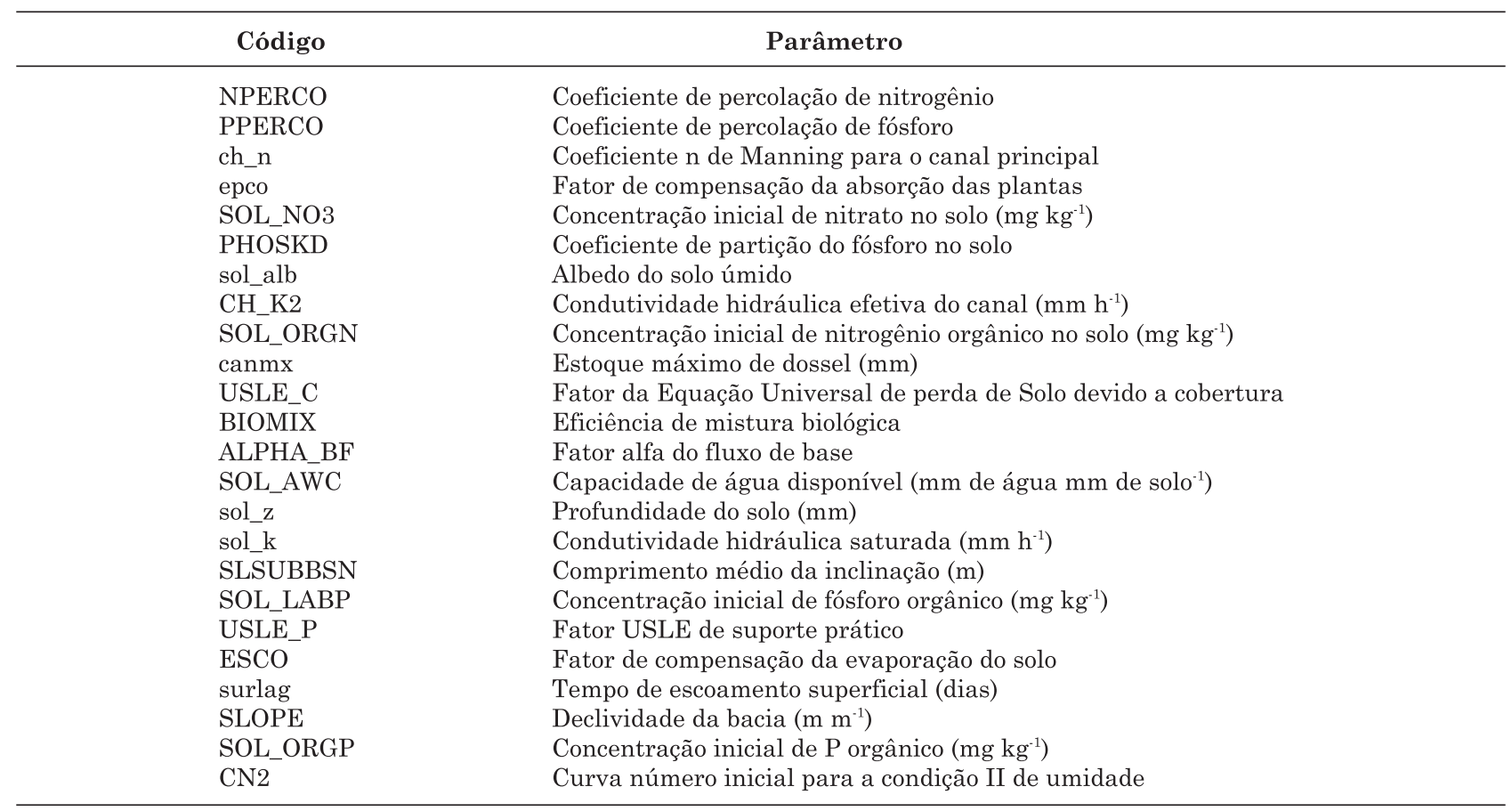




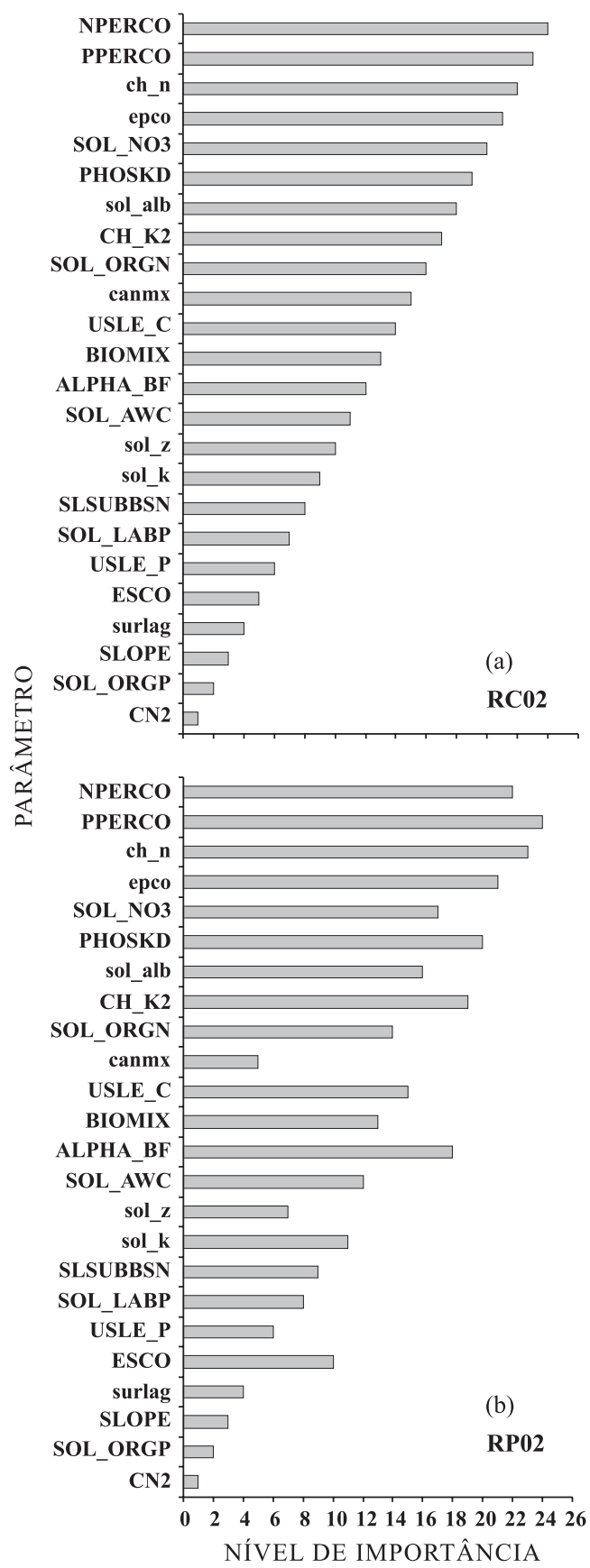

Figura 2. Nível de importância dos parâmetros do modelo, para as estações RC02 (a) e RP02 (b) (códigos do eixo vertical discriminados no quadro 4).

$\mathrm{P}=1.190,10 \mathrm{~kg} \mathrm{ano}^{-1}$, com valor de $C O E=1$, indicando bom ajuste.

Para os dados da estação RP02, a simulação foi realizada para o período de 1/01/2003 a 31/12/2005, com frequência de saída mensal, com 22 registros de dados para sedimentos e $\mathrm{P}$ e 21 registros para vazão. Como a frequência de amostragem foi mensal, porém irregular, para evitar ambiguidades de interpretação pelo SWAT, criou-se um arquivo.txt com dia Juliano, contendo os dados (Griensven, 2007). Para essa estação, foram obtidos resultados das simulações com valores de $C O E$ inferiores a 0,36 , mostrando a inadequação do modelo.

\section{Adição de fósforo ao solo como fertilizante}

O resultado para o cenário empregado foi uma carga de $\mathrm{P}$ total de $3,21 \mathrm{t}^{-1} \mathrm{no}^{-1}$ ou $0,62 \mathrm{~kg} \mathrm{ha}^{-1}$ ano $^{-1}$. Considerando a simulação que completou a calibração, a carga de $\mathrm{P}$ total no exutório foi de $2,89 \mathrm{t} \mathrm{ano}^{-1}$. Portanto, com a adição dos fertilizantes (Quadro 3), ocorreu aumento de $10 \%$ na carga anual de $\mathrm{P}$ total que entra no canal, refletindo qualitativamente a realidade de campo.

Na figura 3 é mostrada a distribuição espacial da exportação de $\mathrm{P}$ total para o cenário empregado.

Observa-se que as sub-bacias a jusante concentram maior carga de $\mathrm{P}$ do que as localizadas na origem dos canais. A carga maior na sub-bacia 12, separada do conjunto formado pelas sub-bacias contíguas ao exutório, deve-se à existência de maior área agrícola contínua. Segundo Reynolds \& Davies (2001), as lavouras aumentam drasticamente as perdas de nutrientes em relação às áreas florestadas.

Na figura 4, observa-se a distribuição mensal da exportação total de $\mathrm{P}$ simulada no exutório da bacia.

As maiores exportações ocorreram nos meses de outubro e novembro, após a aplicação de 135,5 kg de $\mathrm{P}$ como fertilizante nas culturas de milho e soja. Esse valor corresponde a $330,0 \mathrm{~kg} \mathrm{ha}^{-1}$ da fórmula 5-25-25 (milho) e 280,0 kg ha-1 (média das fórmulas 2-18-18 e 2-20-20 para soja).

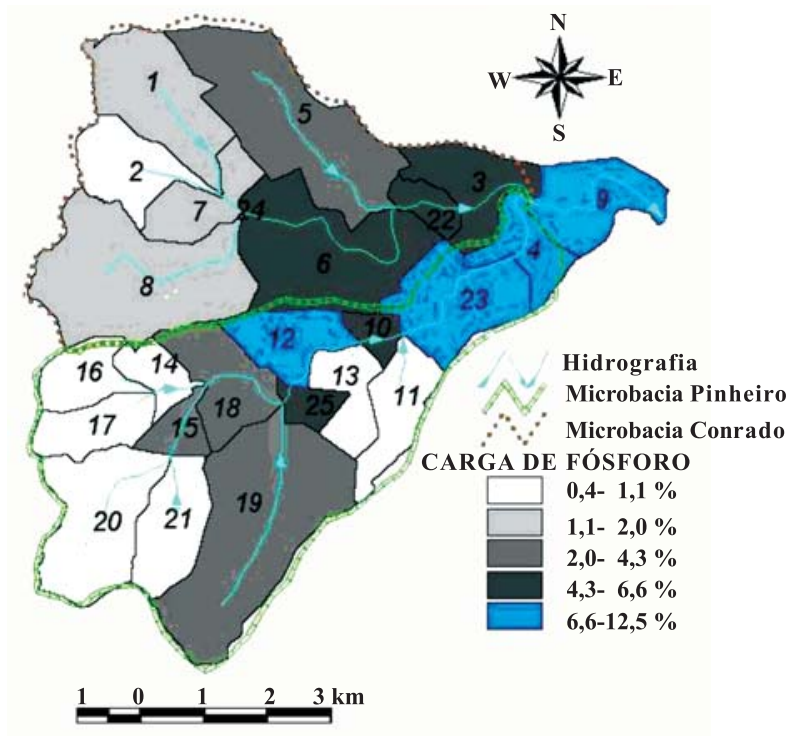

Figura 3. Distribuição espacial da exportação de fósforo total nas microbacias dos rios Conrado e Pinheiro, nos municípios de Pato Branco e Mariópolis (PR). 


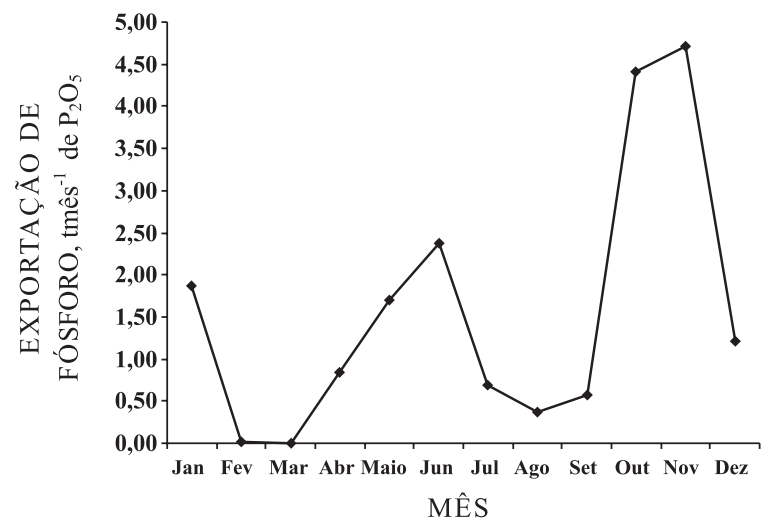

Figura 4. Distribuição mensal da exportação total de fósforo simulada no exutório da bacia.

No mês de junho registra-se o pico resultante da aplicação de cama de aviário na aveia, com valor menor do que o registrado em outubro e novembro. O fato é explicado pela quantidade de $\mathrm{P}$ incorporada à cama de aviário, na qual apenas $1,6 \%$ da matéria seca corresponde ao $\mathrm{P}$ (Palhares, 2004).

\section{CONCLUSÕES}

1. A calibração do modelo SWAT 2005 para a simulação de vazão e exportação de $\mathrm{P}$ total foi facilitada com a realização prévia de análises de sensibilidade.

2. O modelo SWAT 2005 realizou de forma aceitável as simulações de vazão e de exportação de $\mathrm{P}$ total quando a calibração foi feita com dados com frequência de amostragem regular.

3. Quando a frequência de amostragem foi irregular e o número de dados pequeno, os procedimentos de análise de sensibilidade e de autocalibração não foram eficientes na calibração do modelo SWAT 2005 para a simulação de vazão e exportação de $\mathrm{P}$ total.

\section{LITERATURA CITADA}

ARNOLD, J.G. \& FOHRER, N. SWAT 2000: Current capabilities and research opportunities in applied watershed modeling. Hydrol. Process, 9:563-572, 2005.

ARNOLD, J.G.; SRINIVASAN, R.; MUTTIAH, R.S. \& WILLIAMS, J.R. Large area hydrologic modeling and assessment. Part I: Model development. J. Am. Water Res. Association, 34:73-89, 1998.

ASCE. Task Committee on Definition of Criteria for Evaluation of Watershed Models of the Watershed Management. Committee Irrigation and Drainage Division. Criteria for evaluation of watershed models. J. Irrig. Drainage Eng., 119:429-442, 1993.
BENAMAN, J.; SHOEMAKER, C.A. \& HAITH, D.A. Calibration and validation of soil and water assessment tool on an agricultural watershed in upstate New York. ASCE J. Hydrol. Eng., 10:363-374, 2005.

BIESBROUCK, B. \& WYSEURE, G. AVSWAT 2000. Katholieke Universiteit Leuven, Laboratory for Soil and Water Management. Vital Decosterstraat 102, B-3000. Leuven, 2002. 199p.

BITTENCOURT, S. \& GOBBI, E.F. Carga máxima de fósforo admissível ao reservatório Piraquara II, uma aplicação do processo TMDL. R. Bras. Ci. Solo, 30:595-603, 2006.

DI LUZIO, M.; SRINIVASAN, R. \& ARNOLD, J. Arc view interface for SWAT2000 - User's guide. Temple, Blackland Research Center, Texas Agricultural Experiment Station, 2001.

GREEN, C.H. \& GRIENSVEN, A. Autocalibration in hydrologic modeling: Using SWAT 2005 in small-scale watersheds. Environ. Model. Software, 23:422-434, 2008.

GRIENSVEN, A.V. Sensitivity, auto-calibration, uncertainty and model evaluation in SWAT2005. Artigo técnico. Disponível em: <http://groups.google.com/group/swatuser/ files $>$. Acesso em: 16 ago. de 2007.

JAYAKRISHNAN, R.; SRINIVASAN, R.; SANTHI, C. \& ARNOLD, J.G. Advances in the application of the SWAT model for water resources management. Hydrol. Process., 19:749-762, 2005 .

JENSON, S.K. \& DOMINGUE, J.O. Extracting topographic structure from digital elevation data for geographic information system analysis. Photogram. Eng. Rem. Sens., 54:1593-1600, 1988.

LEMONDS, P.J. \& KASCH, M. Comparison of the autocalibration tools available within SWAT 2005 and manual calibration. In: CONFERENCE IN WATERSHED MANAGEMENT TO MEET WATER QUALITY STANDARDS AND TMDLS, 4., San Antonio, 2007. Disponível em: <http://asae.frymulti.com/toc.asp> Acesso em: 27 de outubro de 2007.

MACHADO, R.E. \& VETTORAZZI, C.A. Simulação da produção de sedimentos para a microbacia hidrográfica do Ribeirão dos Marins, SP. R. Bras. Ci. Solo, 27:735-741, 2003 .

MACHADO, W.C.P. Indicadores de qualidade das águas da Bacia Hidrográfica do rio Pato Branco - Sub-bacias dos rios Conrado e Pinheiro. Curitiba, Universidade Federal do Paraná, 2006. 315p. (Tese de Doutorado)

NEITSCH, S.L.; ARNOLD, J.G.; KINIRY, J.R.; WILLIAMS, J.R. \& KING, K.W. Soil and water assessment tool theoretical documentation version 2000. Disponível em: $<$ http://www.brc.tamus.edu/swat/downloads/doc/ swat2000theory.pdf>. Acesso em: 06 de novembro de 2006. 212 p.

NEITSCH, S.L.; ARNOLD, J.G.; KINIRY, J.R.; SPRINIVASAN, R. \& WILLIAMS, J.R. Soil and water assessment tool input/output file documentation version 2005. Disponível em: <http://www.brc.tamus.edu/swat/downloads/doc/ swat2005/SWAT\%202005\%20io.pdf>. Acesso em: 16 de ago. de 2007. 530p. 
PALHARES, J.C.P. Cama de aviário: Possibilidades de utilização. In: Embrapa Suínos e Aves. Artigo técnico. Disponível em: <http://www.cnpsa.embrapa.br/sgc/ sgcartigos/artigosg7c7t6x.html>. Acesso em: 16 de ago. de 2007.

PARANÁ. SUDERHSA. Superintendência dos Recursos Hídricos e Saneamento Ambiental. Programa Nacional de Meio Ambiente II - PNMAII. Disponível em: <http:// www.pr.gov.br/meioambiente/suderhsa/>. Acesso em: 08 de fev. de 2007.

REYNOLDS, C.S. \& DAVIES, P.S. Sources and bioavailability of phosphorus fractions in freshwaters: A British perspective. Biol. Rev., 76:27-64, 2001.
SANTHI, C.; SRINIVASAN, R.; ARNOLD, J.G. \& WILLIAM, J.R. A modeling approach to evaluate the impacts of water quality management plans implemented in a watershed in Texas. Environ. Model. Software, 21:11411157, 2005.

SILVA, P.M.O.; MELLO, C.R.; SILVA, A.M. \& COELHO, G. Modelagem da hidrografa de cheia em uma bacia hidrográfica da região Alto Rio Grande. R. Bras. Eng. Agric. Amb., 12:258-265, 2008. 
